\title{
A Novel Polishing Process with Rigid-Flexible Composite Structure Plate and Its Performance in Polishing Sapphire Wafer
}

\section{Yongchao Xu}

Fujian University of Technology

Chen Lin

Fujian University of Technology

Qianting Wang ( $\square$ wqt@fjut.edu.cn )

Fujian University of Technology

Charlesming Zheng

Fujian University of Technology

\section{Youji Zhan}

Fujian University of Technology

\section{Bingsan Chen}

Fujian University of Technology

\section{Research Article}

Keywords: Sapphire, Flexible polishing, Rigid-flexible composite structure, Surface shape accuracy, Material removal rate

Posted Date: December 28th, 2021

DOI: https://doi.org/10.21203/rs.3.rs-1184854/v1

License: (c) (1) This work is licensed under a Creative Commons Attribution 4.0 International License. Read Full License 


\section{Abstract}

A novel flexible polishing process has been developed for sapphire wafer by using a polishing plate with rigid-flexible composite structure to satisfy the demands of excellent surface shape accuracy and high surface topography quality simultaneously. This new polishing plate was fabricated by alternately casting and curing the ring structure of soft and hard unsaturated resins. It is found that the overall stiffness of the polishing plate is improved due to the "hard support frame" of rigid-flexible polishing plate, as well as the ability of removal selectivity of the polishing plate is strengthened. The topography quality and shape accuracy of sapphire wafer polished by presented novel polishing process have been compared with those polished by conventional flexible polishing, respectively. Both experiment and simulation results are shown that the surface roughness and topographical variations of sapphire wafer polished by the novel rigid-flexible composite structure polishing plate have been greatly improved. Comparing with the conventional flexible polishing, the surface shape accuracy of the sapphire wafer polished by the presented novel polishing process can be improved by $54.1 \%$.

\section{Introduction}

As a typical engineering ceramic material, single crystal sapphire consisting of $\mathrm{a}-\mathrm{Al}_{2} \mathrm{O}_{3}$ has been widely used in many applications such as optics, electronics, and temperature sensing etc., and is the most common wafer used in light emitting diodes (LEDs) by virtue of its excellent mechanical and optical properties such as great hardness, good thermal stability, chemical inertness, and good light transmission[1-4]. The surface quality of processed sapphire wafer plays a critical role in these applications, as well as in shape accuracy. Generally, the processed surface of sapphire wafer is required to be smooth and flat without sub-surface damage to ensure the performance requirements in practice[5-8]. However, as a typical hard-brittle and difficult-to-process material, it is a great challenge for sapphire to satisfy those demands.

Currently, the abrasion processing of sapphire wafer mainly includes slicing, lapping and polishing[9]. The main planarization machining to realize the precision requirement for sapphire wafer is abrasive machining, especially by using abrasive particles with fine grain size[10,11]. A high concentration of slurry that contains relatively large abrasive particles of high hardness, such as diamond and alumina, has been widely used with a metal-resin platen in the conventional mechanical polishing[12, 13]. However, hard abrasives during mechanical polishing lead to poor roughness and heavy scratch on sapphire surface, resulting in a subprime pre-machined surface for next processing step. On the other hand, uneven removal of surface materials can be found due to the uneven dispersion and uncontrollable trajectory of abrasive particles, which lead to the decline of surface shape accuracy. Therefore, it is extremely difficult to obtain fine surface and high surface shape accuracy simultaneously through traditional free abrasive polishing.

In recent years, various reform technologies have been applied to improve the mechanical polishing quality of sapphire wafer, such as fixed abrasive machining[14, 15], mixed abrasive machining[16], 
ultrasonic vibration-assisted machining[17, 18], hydrodynamic machining[19], catalyst-assisted machining[20, 21], composite abrasive machining[22-25]. Although these methods exhibit machining performance superior to the conventional method, some problems associated with the inherent properties of free and fixed abrasive machining still exist. For example, the random distribution and uncontrollable trajectory of the abrasive particles in free abrasive polishing result in the uneven distributed topography and the severe bad of surface shape accuracy. Compared to free abrasive process, fixed abrasive polishing exhibits a significantly worse surface quality, especially a large number of scratches and pits[26].

As a novel super-precision machining process, flexible polishing has been widely used for machining hard and brittle materials as well as various kinds of optical materials, such as $\mathrm{SiC}$, sapphire, and GaN. Flexible polishing is to use the soft binder with yield effect to restrict the movement of abrasive particles within a certain range under certain constraints to form a soft polishing plate. Under a certain polishing pressure, the yielding effect from abrasive polishing plate caused by soft binder is used to make the large-size abrasive particles yield, which enables large-size abrasive particles to achieve relatively high surface quality instead of smaller ones. Therefore, uneven dispersion and uncontrollable trajectory problems of ultrafine abrasives can be effectively avoided. Flexible polishing has been reported as an ideal approach to satisfy the processing demands of scratch-free and nano-scale roughness in wafer surfaces[27]. Yuan et al. [28] prepared a new soft abrasive polishing plate for monocrystalline silicon polishing. Based on the "trap effect" of the polishing plate during processing, the scratches caused by large abrasive particles on the machined wafer can be effectively avoided. Xu et al. [29-31] used sol-gel technology to disperse ultrafine abrasive evenly in sodium alginate solution, and then solidified with $\mathrm{Ca}^{2+}$ solution to form semi-fixed flexible polishing plate. This technology was proposed for the mechanical polishing of single crystal sapphire, single crystal silicon carbide and other photoelectric wafer materials to achieve nano-scale surface roughness. Bai et al. [32] used the constrained abrasive particle effect of magnetorheological polishing to achieve ultra-smooth and low damage polishing of hard and brittle materials, significantly reducing or even eliminating the damage caused by large-size abrasive particles to the machined surface. The above research results show that compared with the traditional free abrasive and fixed abrasive polishing, using the flexible polishing plate with hard abrasive to process the wafer material can obtain a smoother and less sub surface damage surface. However, in the process of wafer polishing, it is not enough to only obtain better surface quality, but also need higher surface shape accuracy. Due to the surface of flexible polishing plate is relatively soft, it will lead to certain deformation of polishing plate under the action of polishing pressure, which is difficult to ensure the surface shape accuracy of wafer processing. Therefore, it is essential to develop a new method to meet the perfect surface and high surface shape accuracy of sapphire wafer polishing at the same time.

In this study, we introduce a novel flexible polishing by using rigid-flexible composite structure polishing plate (RCSPP), and investigated its polishing performance. In the RCSPP, the rigid structural material without any abrasive is used as the "support frame", which can reduce the deformation of the polishing plate and ensure the surface shape accuracy in the polishing process. Meanwhile, the soft structural 
material is used as the "processing layer" to realize the flexible polishing of the wafer and obtain better wafer surface quality. A conventional flexible polishing is performed for comparative study. The surface topography and shape accuracy of sapphire wafer after the flexible polishing with different plates are performed, and the MRR of flexible polishing process is measured. It will be shown that using this polishing process instead of conventional flexible polishing, smoother surface and high surface shape accuracy can be obtained at the same time. Furthermore, through finite element analysis, the effect of rigid structure on surface shape accuracy is discussed in detail.

\section{Materials And Experimental Methods}

\subsection{Manufacturing procedures of polishing plates}

The manufacturing procedures of RCSPP via casting technique mainly includes seven procedures, as shown in Fig. 1: (a) promote agent and curing agent are successively added into the hard unsaturated resin solution and stirred evenly by thermostatic mixer; (b) the hard unsaturated resin solution was poured on the annular groove, which is pasted on the glass plate; (c) wait for the hard unsaturated resin solution to cure for 4 hours; (d) only the acrylic plates are removed; (e) promote agent, alumina abrasives (the average particle size is $40 \mu \mathrm{m}$ ) and curing agent are successively added into the soft unsaturated resin solution in and stirred uniformly; ( $f$ ) wait for the soft unsaturated resin solution to cure for 4 hours to form RCSPP; (g) take out the RCSPP with the turnover formwork. Moreover, the conventional flexible polishing plate is made from soft unsaturated resin by mixing, screeding and curing processes, as shown in Fig. 1(e) and (h).

\subsection{Polishing tests}

Commercial lapping-machined sapphire wafer-oriented $\left(\begin{array}{llll}0 & 0 & 0 & 1\end{array}\right)$ plane with two-inch diameter was used, and its original surface roughness Ra was about $1 \mu \mathrm{m}$. Polishing experiments were conducted on a rotary-type polishing machine (AUTOPOL-1200S, Kejing, China) with conventional flexible polishing plate and RCSPP, respectively. Deionized water was applied as the coolant. The schematic diagram of the sapphire wafer polishing process is shown in Fig. 2.

The acting force between the wafer carrier and the polishing plate was $5 \mathrm{kgf}$. The workpiece/plate rotation speed was $60 / 120 \mathrm{rpm}$, and the processing time was $120 \mathrm{~min}$. After processing, the wafers were cleaned immediately with ethanol and deionized water under sonication. The weight of the wafers before and after polishing was measured by a precision electronic balance with $0.01 \mathrm{mg}$ precision (GE0505, Yoke, China) to calculate the MRR according to the following formula[33]: 


$$
M R R=\frac{10^{7} \times \Delta m}{\rho \times 2.54^{2} \times \pi \times t}
$$

Where $\Delta m(\mathrm{mg})$ is the mass loss of sapphire wafer before and after polishing, $t(\mathrm{~min})$ is polishing time, $\rho$ is sapphire density $\left(3.98 \mathrm{~g} / \mathrm{cm}^{3}\right)$, and $M R R(\mathrm{~nm} / \mathrm{min})$ is the corresponding material removal rate.

The surface profile and roughness of processed sapphire wafers were evaluated using a contact roughness instrument (MarSurf XR20, Mahr, Germany). For each machined wafer, nine testing points roughness $R$ a that evenly dispersed on the wafer surface were conducted at different locations, as illustrated in Fig. 3, and the average roughness Ra was calculated. The standard deviation (SD) was calculated by Eq. (2) using nine testing points thickness changes (the same testing points as mentioned above) measured by a digital micrometer (ACE-G3121, Rapid measurement, China), which was used to evaluate the surface shape accuracy. The $S D$ can be expressed as:

$$
S D=\sqrt{\frac{\sum_{i=1}^{n}\left(X_{\mathrm{i}}-\bar{X}\right)^{2}}{n-1}}
$$

where $n$ is the total number of testing points, $X_{i}$ is the ith testing point value, $\bar{X}$ is average of all testing point values.

Moreover, the compression tests of rigid and flexible materials were carried out on a universal testing machine of mechanics of materials (2382, Instron, USA) to obtain the mechanical properties parameters of materials used for simulation.

\section{Effect Of Rigid Structure On Surface Shape Accuracy}

In order to study the effect of rigid structure of rigid-flexible composite plate on surface shape accuracy, stress and displacement between different polishing layers and sapphire wafer surface were performed by finite element analysis. Abaqus finite element program (Version 6.14, Dassault Systemes Simulia Corp., Providence, R.I.) is used to analyze the material removal process during conventional flexible and RCSPP polishing from a microscopic point of view.

On the whole, due to the surface of sapphire wafer is uneven and continuous[34], assuming the local area of sapphire wafer surface is a unidirectional curved surface. The local area of contact interface between polishing plate and workpiece is taken as the geometrical and mesh model as shown in Fig. 4. The sapphire wafer and polishing plate are established by solid element type for the authenticity of the geometrical model. In order to improve the calculate accuracy, hexahedron mesh is used on sapphire wafer and polishing plate. The number of divisiory unit of sapphire wafer is 2700 , the number of rigid area and flexible area of RCSPP's divisiory unit are 4500 respectively (Fig. 4a), the number of the conventional flexible polishing plate's divisiory unit is 9000 (Fig. 4b). The mechanical property 
parameters of sapphire wafer, rigid material and flexible material are listed in Table 1. In terms of boundary conditions, sapphire wafer is loaded in the $Y$ direction and constrained by three rotational degrees of freedom and two displacement degrees of freedom of $X$ and $Z$ axis and bottom of the polishing plate is completely fixed.

Table 1

Mechanical property parameters of sapphire and polishing plate materials.

\begin{tabular}{|lll|}
\hline Material & Young's Modulus / MPa & Poisson's Ratio \\
\hline Sapphire[35] & 385000 & 0.22 \\
\hline Rigid material & 933.75 & 0.38 \\
\hline Flexible material & 13.45 & 0.35 \\
\hline
\end{tabular}

The simulation results of local stress and displacement at the processing interface between sapphire wafer surface and different polishing layers at different positions are shown in Fig. 5. For the conventional flexible polishing, as shown in Fig. 5(a), when the sapphire wafer interacts with the conventional flexible polishing plate, the flexible structural materials containing abrasives will undergo elastic deformation under the action of polishing pressure, resulting in the processing area adhering to the whole non-plane of the sapphire wafer, thus reducing the surface shape accuracy of the sapphire wafer. Meanwhile, following five cases of material removal process during RCSPP polishing are addressed:

1) When the local highest point of wafer surface contacts with the rigid structural materials of RCSPP at the position shown in Fig. 5(b), it can be seen that under the action of polishing pressure, the stress and displacement change area almost only occurs in the rigid structural materials without any abrasive, while at this time, the flexible structural material with hard abrasives has no contact with the wafer surface. Therefore, the polishing plate only supports the sapphire wafer at this position without any material removal.

2) When the local highest point of wafer surface contacts with the rigid structural materials of RCSPP at the position shown in Fig. 5(c), the stress area is similar to that in Fig. 5(b), and the flexible structural material of the polishing plate begins to deform slightly due to the small deformation of the rigid material, and the material is rarely removed near the local highest point.

3) When the local highest point of the sapphire wafer contacts the intersection of rigid and flexible structural materials of RCSPP, as shown in Fig. 5(d), the local highest point of sapphire wafer contacts with the soft and hard areas simultaneously. Due to the different hardness of two unsaturated resins, the flexible structural material is relatively less stressed under the support of the rigid structural material, and its flexibility makes the displacement change greatly. Meanwhile, under the support of the rigid structural material without any abrasive, the flexible structural material containing abrasives being to slightly remove the wafer material near the local highest point. 
4) When the local highest point of wafer surface contacts with the flexible structural materials of RCSPP at the position shown in Fig. 5(e), the stress diagram shows that when the local highest point of sapphire wafer reaches the limit of the soft region, the stress of the rigid structural materials is the largest at the boundary line, which supports the sapphire wafer. Locally machining surface is divided into processing area and non-processing area by the position of the support, in which the upper location of the support is a non-processing area, and the lower location of the support is a processing area in contact with the soft area with abrasives.

5) When the local highest point of wafer surface contacts with the flexible structural materials of RCSPP at the position shown in Fig. 5(f), under the support action of rigid structure, the local highest point of the sapphire wafer move to the location with maximum displacement in flexible structural materials, and the contact arc length between wafer surface and flexible structural materials reaches the maximum, as well as the material remove.

Based on the above analysis, the existing of rigid structure in RCSPP makes the flexible structural materials with abrasives obtain the ability of removal selectivity, which means that the material in the convex area of the wafer surface will be removed preferentially. On the contrary, the material in the concave area will be removed as the convex area is planarized. Based on this characteristic, the novel flexible polishing with RCSPP can effectively improve the surface shape accuracy in the polishing of sapphire wafer.

\section{Results And Discussion}

\subsection{Comparison of planarization behavior}

In order to compare the planarization behavior of different flexible polishing processes, the wafers were polished using conventional and RCSPP polishing after lapping process. Two variations of surface roughness $R$ a of sapphire wafer after two kinds of flexible polishing are shown in Fig. 6 . Under the yielding effect of flexible material, it is observed that the wafer surface roughness Ra decreases from about $1 \mu \mathrm{m}$ to $0.7 \mu \mathrm{m}$ after four polishing intervals in conventional flexible polishing. Meanwhile, the surface roughness of sapphire wafer processed by RCSPP polishing is similar to that of the conventional flexible polishing under the same machining parameters, and the surface roughness of sapphire wafer is reduced by nearly $0.3 \mu \mathrm{m}$.

Figure 7 shows the surface profile curves of sapphire wafers before and after polishing. As shown in Figure 7(a), lapping-machined sapphire wafer surface exhibits a large number of deep scratches and irregularity on the surface of sapphire wafer. These scratches could also cause an obvious increase of roughness. In terms of the above results, both of the two flexible polishing approaches could effectively smooth the surface as presented in Figure 7(b) and (c). While the great smoother surface acquired from the flexible polishing by using conventional and RCSPP polishing, the processed wafers have a higher definition than original surface, and the vast majority of scratches are removed as shown in Figure 7(b) 
and (c). It can also be shown that the value of PV (peak-to-valley) is $>9 \mathrm{~nm}$ before polishing, and $<5 \mathrm{~nm}$ after polishing. Both kinds of flexible polishing can significantly reduce the scratch depth of sapphire wafer. These results show that the surface roughness polished by rigid-flexible composite structure polishing is consistent with that obtained by conventional flexible polishing, indicating that the rigid structural in novel flexible polishing will not reduce the planarization ability of the polishing plate.

\subsection{Comparison of surface shape accuracy}

Two kinds of flexible polishing are used to compare the surface shape accuracy of polished sapphire wafer, as shown in Fig. 8. It can be seen that with the extension of polishing time, the surface shape accuracy of sapphire wafer processed by novel flexible polishing with RCSPP has been significantly improved. Comparing with the conventional flexible polishing, the surface shape accuracy of the sapphire wafer polished by the presented novel polishing process can be improved by $54.1 \%$. Due to the existence of "hard support frame" of rigid structural materials in the novel polishing process, which cause the overall stiffness of the polishing plate is improved as well as obtained the ability of removal selectivity, the surface flatness error of the polishing plate in the polishing process is dramatically reduced. Therefore, the use of RCSPP can significantly improve the surface shape accuracy of processed wafer.

\subsection{Comparison of $M R R$}

Figure 9 shows the MRR of sapphire wafers processed by conventional flexible and RCSPP polishing. It can be seen that the MRR was affected by the rigid structure in RCSPP. As presented in Fig. 8, the material removal rate of sapphire wafer polished by RCSPP polishing is $1.2 \mathrm{~nm} / \mathrm{min}$ lower than that of the conventional flexible polishing. It can be explained by that the presence of rigid structural materials without any abrasive, the effective number of abrasive particles in contact with sapphire wafer surface is reduced. These results indicate that the material removal of sapphire wafer is affected by the rigid structure, but its quantitative relationship needs further investigation.

\section{Conclusions}

In this study, a novel flexible polishing process has been presented with RCSPP to meet the demands of excellent surface accuracy and high surface topography quality simultaneously for sapphire wafer. The conventional flexible and RCSPP polishing have been performed for comparative study. Meanwhile, combined with finite element analysis, the effects of rigid structure on surface shape accuracy have been discussed in detail. The following conclusions can be drawn as:

1. The rigid structure can greatly improve the surface shape accuracy of sapphire wafer in proposed novel flexible polishing process. Comparing with the conventional flexible polishing, the surface shape accuracy of the sapphire wafer polished by the presented novel polishing process can be improved by $54.1 \%$. Meanwhile, it is found that the surface of sapphire wafer polished by novel 
flexible polishing has the same surface planarization ability, surface roughness and topographical variation as the conventional flexible method, but the $M R R$ will be reduced accordingly.

2. Due to the existence of rigid structural in RCSPP, on the one hand, the overall stiffness of polishing plate is improved and the deformation of the polishing plate in the polishing process is reduced; On the other hand, it enables the flexible abrasive materials to obtain the ability of removal selectivity. The materials in the convex area of the wafer surface will be removed preferentially, and the materials in the concave area will be removed with the planarization of the convex area.

\section{Declarations}

\section{Funding}

The authors appreciate financial supports from Program for Innovative Research Team in Science and Technologe in Fujian Province University (IRTSTFJ), National Natural Science Foundation of China (Grant No. 51775113), Educational and Scientific Research Program for Young and Middle-aged Teachers of the Education Department of Fujian Province (Grant No. JAT190402) and Fujian University of Technology scientific research fund (Grant No. GY-Z19124).

\section{Competing Interests}

The authors have no relevant financial or non-financial interests to disclose.

\section{Conflicts of interest}

The authors declare that they have no conflict of interest.

Availability of data and materialnNot applicable

Code availability: Not applicable

Ethics approval: Not applicable

Consent to participate: Not applicable

Consent for publication: Not applicable

\section{Author Contributions}

All authors contributed to the study conception and design. Yongchao Xu and Chen Lin contributed significantly to the compilation of experimental results and wrote the first draft of the manuscript. Qianting Wang and Charlesming Zheng helped analyze and revise the manuscript. Youji Zhan and Bingsan Chen contributed in the final proofreading of the review manuscript.

\section{References}


[1] Liu HK, Chen CCA, Chen WC (2020) Effects of compound diamond slurry with graphene for lapping of sapphire wafers. Int J Adv Manuf Tech 106: 4755-4768

[2] Huang SQ, Li XL, Zhao YT, Sun Q, Huang H (2021) A novel lapping process for single-crystal sapphire using hybrid nanoparticle suspensions. Int J Mech Sci 191: 106099

[3] YV Klunnikova, MV Anikeev, AV Filimonov, R Kumar (2020) Machine learning application for prediction of sapphire crystals defects. J Electron Sci Technol 18: 100029

[4] DV Ananchenko, SV Nikiforov, VN Kuzovkov, Al Popov, GR Ramazanov, RI Batalov, RM Bayazitov, HA Novikov (2020) Radiation-induced defects in sapphire single crystals irradiated by a pulsed ion beam. Nucl Instrum Methods Phys Res Sect B 466: 1-7

[5] KJ Lee, S Oh, SJ Kim, SY Yim, N Myoung, K Lee, JS Kim, SH Jung, TH Chung, SJ Park (2019) Enhanced optical output in InGaN/GaN light-emitting diodes by tailored refractive index of nanoporous GaN. Nanotechnology 30: 415301

[6] ZQ L, XB Wang, YB Wu, LJ Xie, L Jiao, WX Zhao (2013) Experimental study on brittle-ductile transition in elliptical ultrasonic assisted grinding (EUAG) of monocrystal sapphire using single diamond abrasive grain. Int J Mach Tool Manu 71: 41-51

[7] Y Li, WL Wang, XC Li, LG Huang, ZT Lin, YL Zheng, XF Chen, GQ Li (2019) Stress and dislocation control of GaN epitaxial films grown on Si substrates and their application in high-performance lightemitting diodes. J Alloy Compd 771: 1000-1008

[8] QC Nie, ZM Jiang, ZY Gan, S Liu, H Yan, HS Fang (2018) Defect analysis of the LED structure deposited on the sapphire substrate. J Cryst Growth 488: 1-7

[9] ZC Li, ZJ Pei, PD Funkenbusch (2011) Machining processes for sapphire wafers: a literature review. Proc Inst Mech Eng Part B 225: 975-989

[10] L Xu, H Lei, TX Wang, Y Dong, SW Dai (2019) Preparation of flower-shaped silica abrasives by double system template method and its effect on polishing performance of sapphire wafers. Ceram Int 45: 84718476

[11] D Yin, XH Niu, K Zhang, JC Wang, YQ Cui (2018) Preparation of MgO doped colloidal $\mathrm{SiO}_{2}$ abrasive and their chemical mechanical polishing performance on c-, $r$ - and a-plane sapphire substrate. Ceram Int 44: 14631-14637

[12] B Luo, QS Yan, JS Pan, JB Lu, ZL Huang (2021) Influences of processing parameters on metalbonded diamond wheel wear when grinding a sapphire wafer. Diam Relat Mater 113: 108275

[13] QF Luo, J Lu, XP Xu (2016) Study on the processing characteristics of SiC and sapphire substrates polished by semi-fixed and fixed abrasive tools. Tribol Int 104: 191-203 
[14] JP Chen, YW Zhu, JB Wang, YN Peng, JG Yao, S Ming (2019) Relationship between mechanical properties and processing performance of agglomerated diamond abrasive compared with single diamond abrasive. Diam Relat Mater 100: 107595

[15] ZL Ju, YW Zhu, JB Wang, DW Zuo (2015) The effect of different slurries on material removal of sapphire surface in fixed-abrasive lapping. Int J Mach Mach Mater 17: 39-48

[16] YC Xu, J Lu, XP Xu (2016) Study on planarization machining of sapphire wafer with soft-hard mixed abrasive through mechanical chemical polishing. Appl Surf Sci 389: 713-720

[17] QY Wang, ZQ Liang, XB Wang, TF Zhou, WX Zhao, YB Wu, L Jiao (2016) Investigation on surface formation mechanism in elliptical ultrasonic assisted grinding (EUAG) of monocrystal sapphire based on fractal analysis method. Int J Adv Manuf Tech 87: 2933-2942

[18] WH Xu, XC Lu, GS Pan, YZ Lei, JB Luo (2010) Ultrasonic flexural vibration assisted chemical mechanical polishing for sapphire substrate. Appl Surf Sci 256: 3936-3940

[19] P Kumar, S More, R Singh, SS Joshi (2013) Experimental characterization of plane and conformal hydrodynamic polishing of machined single crystal sapphire. Manuf Lett 1: 70-73

[20] YC Xu, J Lu, XP Xu (2019) Pollution-Free Approaches for highly efficient sapphire substrate processing by mechanical chemical polishing. Catalysts 7: 594

[21] L Xu, X Zhang, CX Kang, RR Wang, CL Zou, Y Zhou, GS Pan (2018) Preparation of a novel catalyst (SoFellI) and its catalytic performance towards the removal rate of sapphire substrate during CMP process. Tribol Int 120: 99-104

[22] TX Wang, $\mathrm{H}$ Lei (2019) Novel polyelectrolyte- $\mathrm{Al}_{2} \mathrm{O}_{3} / \mathrm{SiO}_{2}$ composite nanoabrasives for improved chemical mechanical polishing (CMP) of sapphire. J Mater Res 3: 1073-1082

[23] H Lei, TT Liu, L Xu (2019) Synthesis of Sm-doped colloidal $\mathrm{SiO}_{2}$ composite abrasives and their chemical mechanical polishing performances on sapphire substrates. Mater Chem Phys 237: 121819

[24] Y Dong, H Lei, WQ Liu, Y Chen (2019) Preparation of ellipsoidal rod-shaped silica nanocomposite abrasives by Chromium ion/PEG200 induced method for sapphire substrates chemical mechanical polishing. J Alloy Compd 777: 1294-1303

[25] TT Liu, H Lei (2017) $\mathrm{Nd}^{3+}$-doped colloidal $\mathrm{SiO}_{2}$ composite abrasives: Synthesis and the effects on chemical mechanical polishing (CMP) performances of sapphire wafers. Appl Surf Sci 413: 16-26

[26] HM Kim, GH Park, YG Seo, DJ Moon, BJ Cho, JG Park (2015) Comparison between sapphire lapping processes using 2-body and 3-body modes as a function of diamond abrasive size. Wear 332-333: 794799 
[27] YC Xu, J Lu, XP Xu, CCA Chen, YJ Lin (2018) Study on high efficient sapphire wafer processing by coupling SG-mechanical polishing and GLA-CMP. Int J Mach Tool Manu 130-131: 12-19

[28] JL Yuan, ZW Wang, T Hong, QF Deng, DH Wen, BH Lv (2009) A semi-fixed abrasive machining technique. J Micromech Microeng 19: 54006

[29] J Lu, YC Xu, YH Zhang, XP Xu (2017) The effects of $\mathrm{SiO}_{2}$ coating on diamond abrasives in sol-gel tool for $\mathrm{SiC}$ substrate polishing. Diam Relat Mater 76: 123-131

[30] J Lu, Y Li, XP Xu (2015) The effects of abrasive yielding on the polishing of SiC wafers using a semifixed flexible pad. Proc Inst Mech Eng Part B 229: 170-177

[31] J Liu, XP Xu (2004) Fabrication of ultra-fine diamond abrasive tools by Sol-Gel. Mater Sci Forum 471472: $426-430$

[32] ZW Bai, QS Yan, JB Lu, XP Xu (2014) Parametric investigation into accommodate-sinking effect of cluster magnetorheological effect pad. Int J Adv Manuf Tech 75: 1447-1456

[33] ZF Zhang, WX Yan, L Zhang, WL Liu, ZT Song (2011) Effect of mechanical process parameters on friction behavior and material removal during sapphire chemical mechanical polishing. Microelectron Eng 88: 3020-3023

[34] CF Fang, C Liu, ZX Zhao, YF Lin, ZW Hu, XP Xu (2018) Study on geometrical patterns of textured fixed-abrasive pads in sapphire lapping based on trajectory analysis. Precis Eng 53: 169-178

[35] L Godino, I Pombo, J Girardot, JA Sanchez, I lordanoff (2020) Modelling the wear evolution of a single alumina abrasive grain: Analyzing the influence of crystalline structure. J Mater Process Technol 277: 116464

\section{Figures}




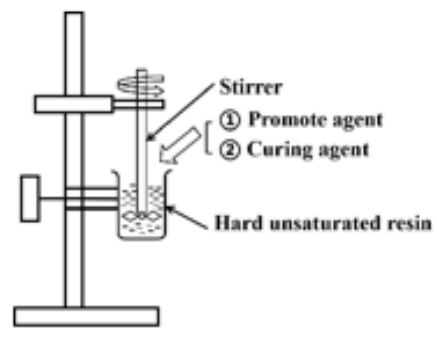

(a) Mixing

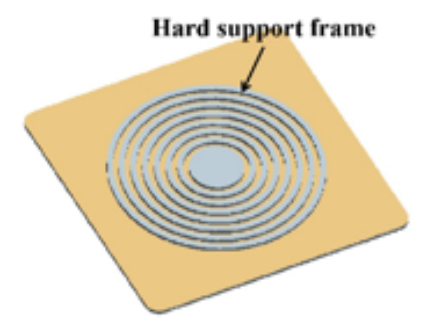

(d) Taking

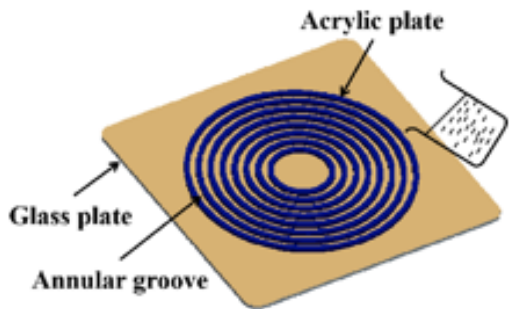

(b) Casting

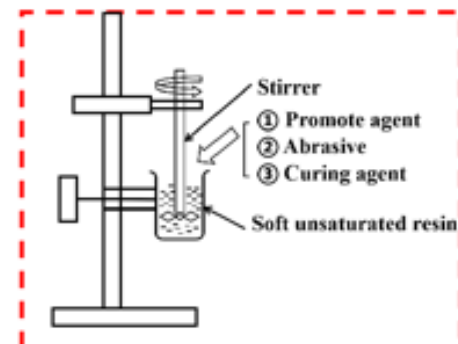

(e) Mixing

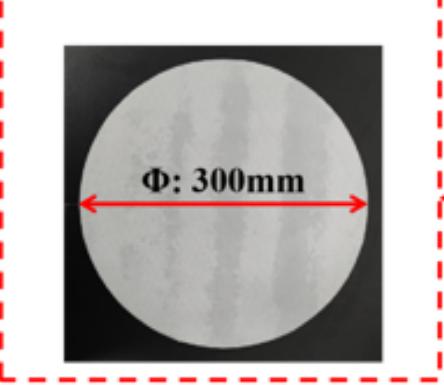

(h) $\overline{\text { Conventional flexible }}$ polishing plate

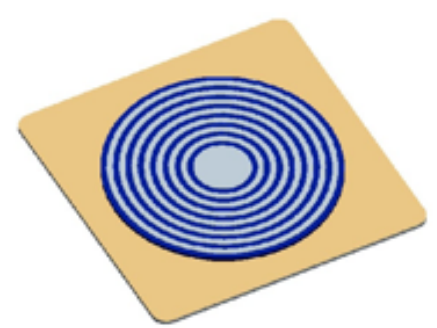

(c) Curing

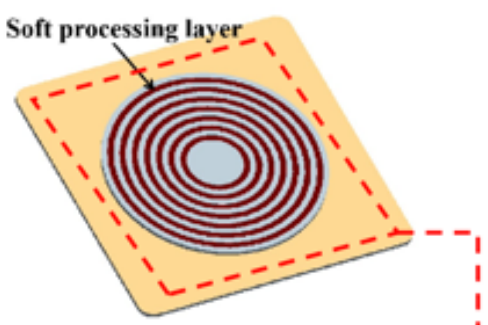

(f) Curing

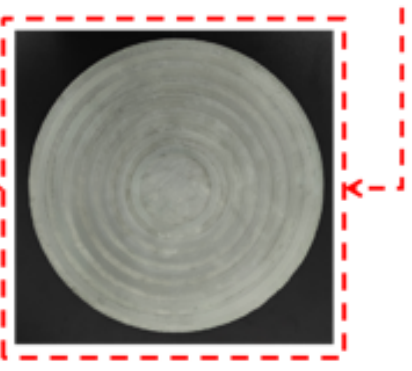

(g) RCSPP

\section{Figure 1}

Schematics of the manufacturing procedures of conventional flexible polishing plate and RCSPP. 


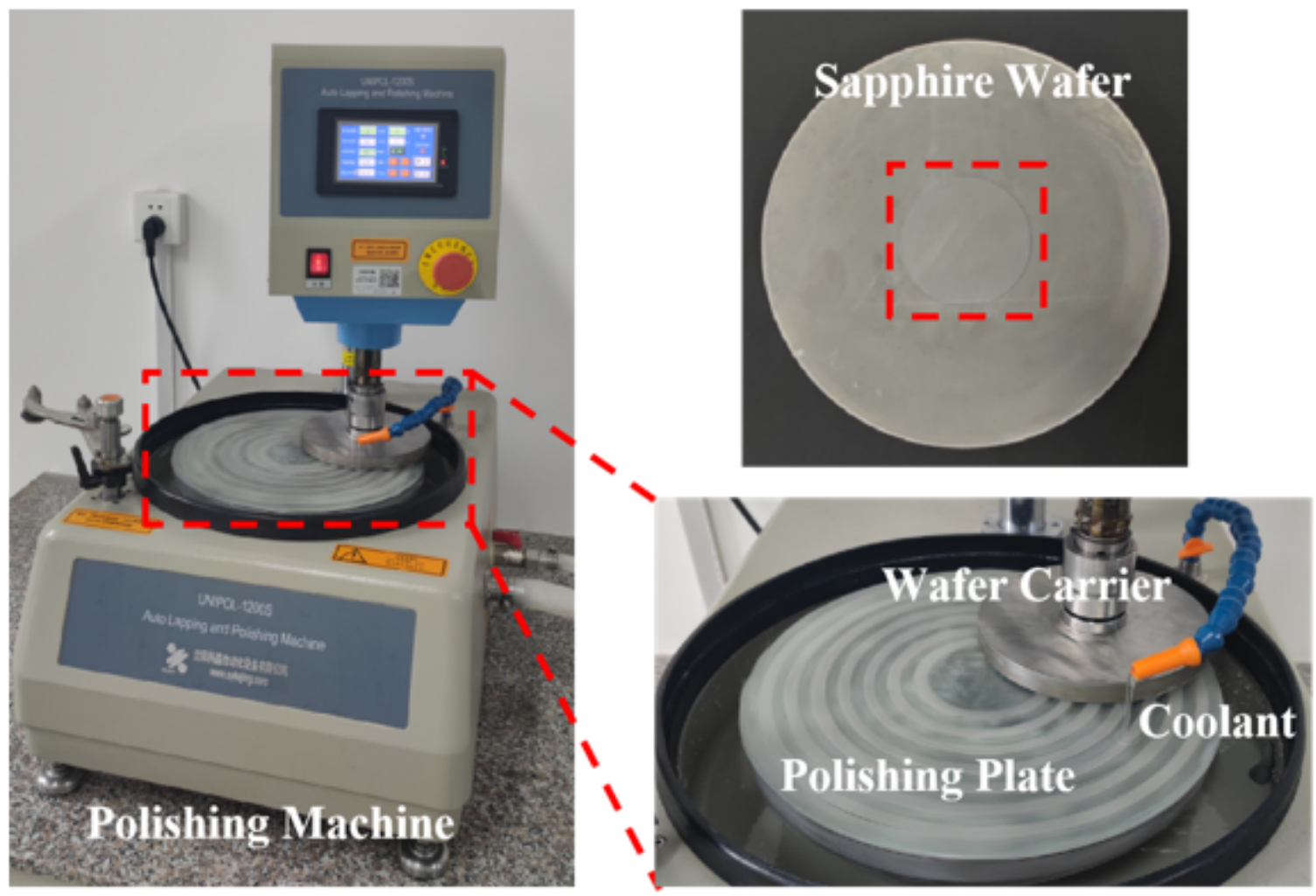

Figure 2

Schematic diagram of the rotary-type polishing experimental setup.

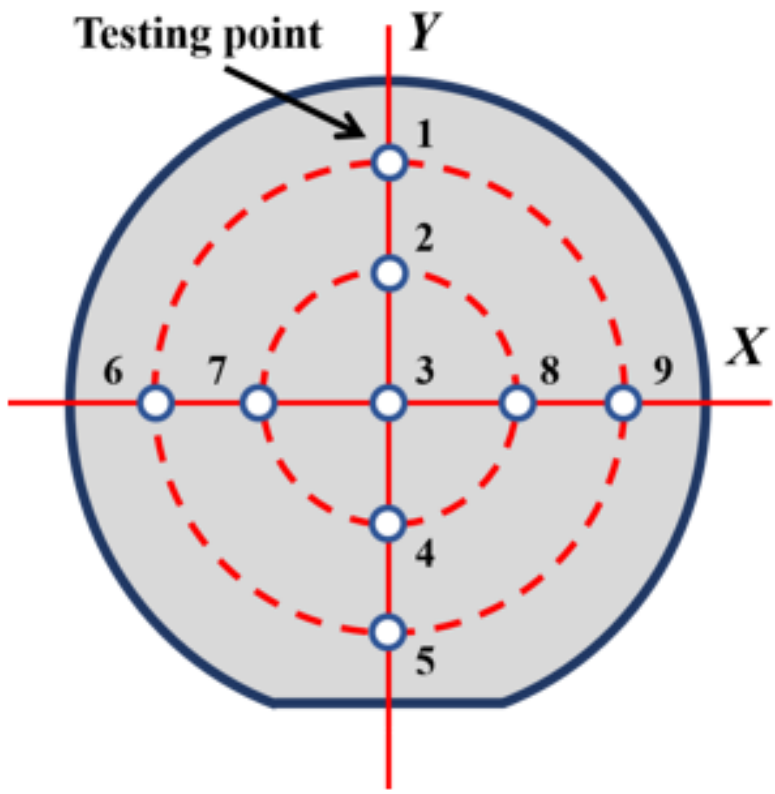


Figure 3

Testing points of surface roughness and thickness change.

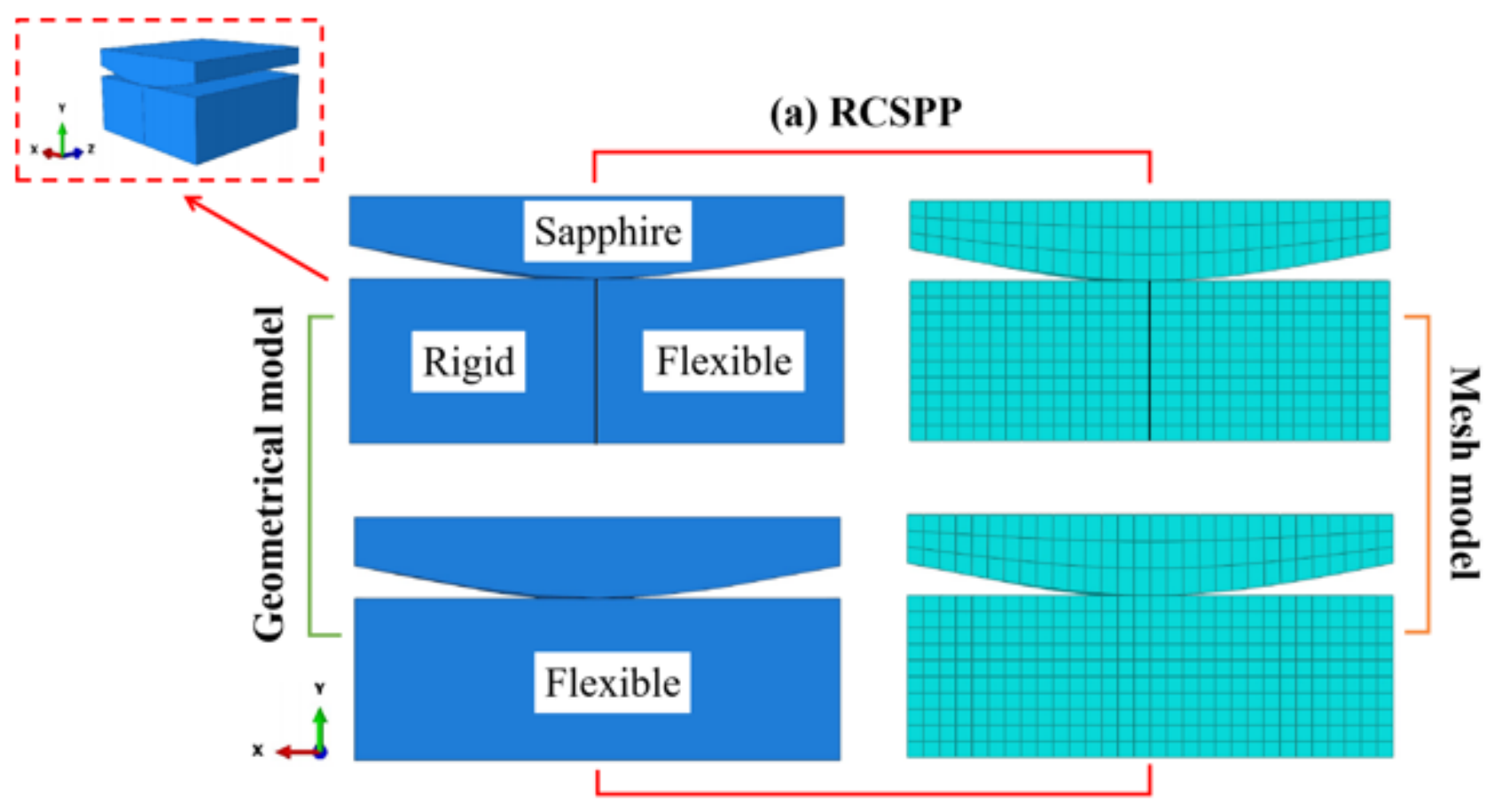

(b) Conventional flexible polishing plate

Figure 4

Geometrical and mesh model of the local area of contact interface between polishing plate and workpiece.

\section{Figure 5}

Stress and displacement at the local action area between different polishing plates and sapphire wafer surface.

\section{Figure 6}

The surface roughness $R a$ of sapphire wafer after two kinds of flexible polishing. 
(a)

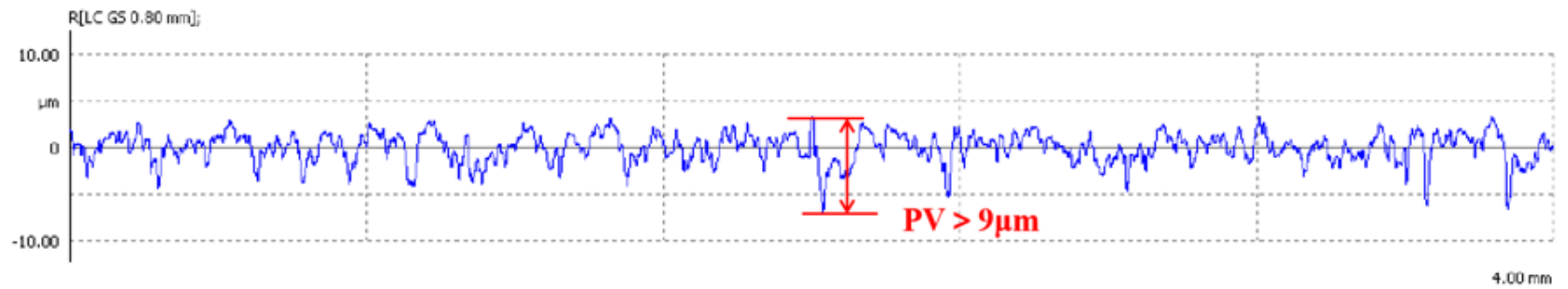

(b)

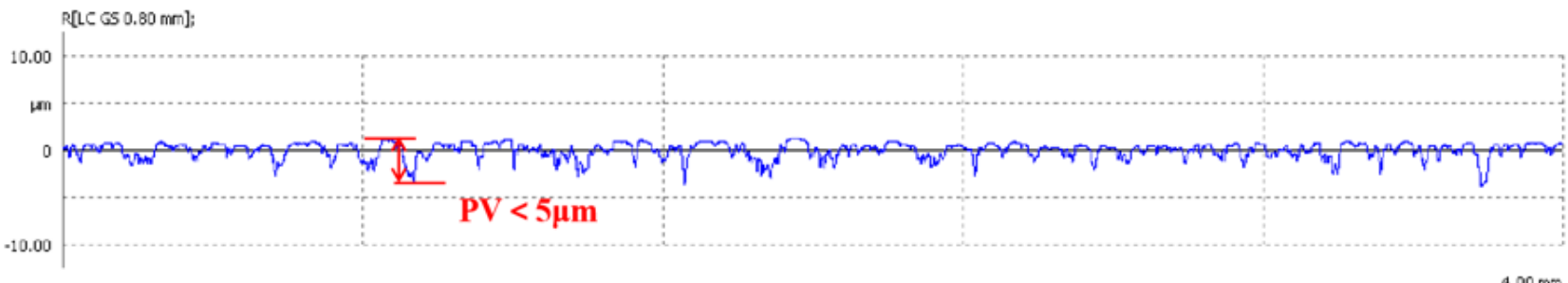

(c)

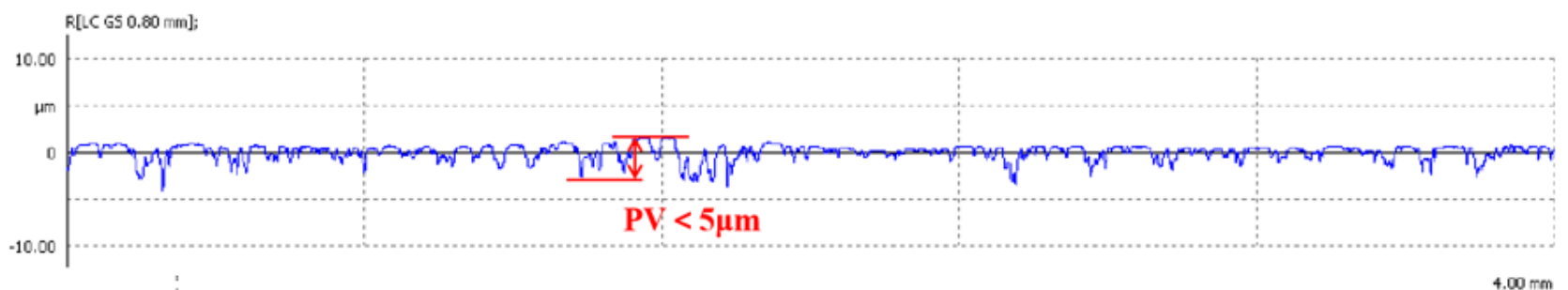

\section{Figure 7}

Surface profile curves of sapphire wafers: (a) before polishing, polished by (b) conventional flexible and (c) RCSPP polishing. 


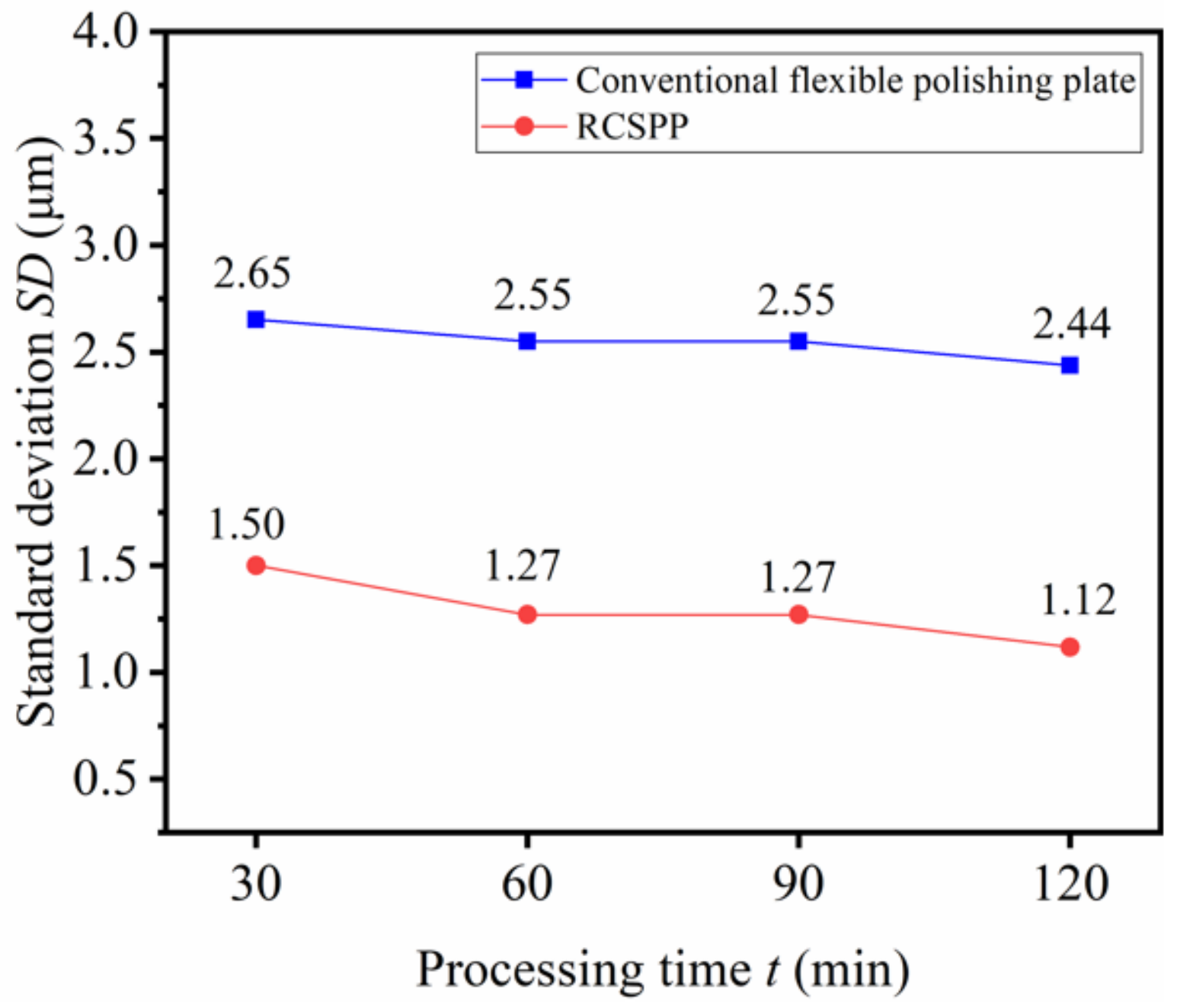

Figure 8

Surface shape accuracy of sapphire wafers processed by conventional flexible and RCSPP polishing. 


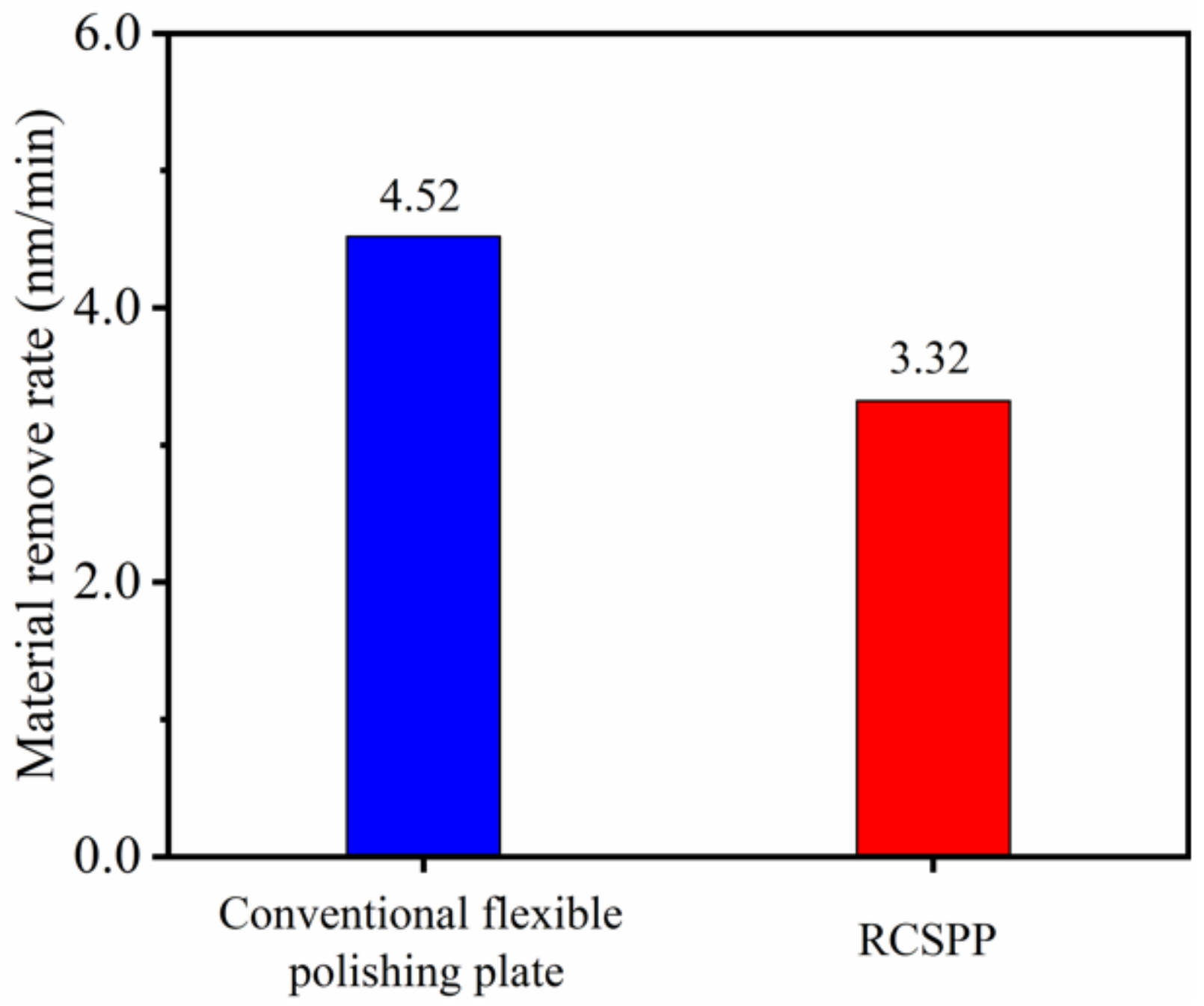

Figure 9

MRR of sapphire wafer processed by polished by conventional and RCSPP polishing. 